or $\mathrm{SD}(1.49 \%, \mathrm{p}=0.999)$ groups. In the Cox proportional analysis, hazard ratios of time to first RSVH in CF were similar to MD $(\mathrm{p}=0.272)$ and SD infants $(\mathrm{p}=0.422)$.

Conclusions This is the largest report of CF infants who have received palivizumab world-wide. Despite RI rate differences, RSVH rates appear similar to those in MD and SD.

\section{IMPACT ANALYSIS OF AN EVIDENCE BASED GUIDELINE ON URINARY TRACT INFECTION (UTI) IN CHILDREN: DETERMINANTS OF IMPLEMENTATION}

doi:10.1136/archdischild-2012-302724.0264

D Geurts, W Vos, H Moll, R Oostenbrink. Paediatrics, Erasmus MC - Sophia Children's Hospital, Rotterdam, The Netherlands

Background To improve reliable diagnosing UTI in young, nontoilet trained children, a diagnostic strategy of the Nice guideline was nationally introduced in the Netherlands. This study aims to determine the impact of this new diagnostic strategy in clinical practice. We evaluated determinants of successful implementation. Methods We conducted a cross-sectional observational study, with observations before and after implementation. We prospectively collected data from healthy children aged 1 month- 2 years, presenting with fever at the emergency room at Sophia Children's Hospital in 2008 and 2010-2011. Primary outcome measure: assessment of children suspected with UTI according to the guideline and determinants of implementation. Secondary outcomes: number of contaminated cultures, hospitalisation and antibiotic treatment. Outcome measures are assessed by mean (95\%CI). Differences before and after implementation were tested using Chi-squared test. Effects of determinants were evaluated using regression analysis.

Results The preintervention group consisted of 207 children (male $64.3 \%$, median age 0.98 years (interquartile range IOR0.77), the postintervention group 194 children (male $55.2 \%$, median age 1.06 year (IOR0.78). Correctly diagnosed UTI increased from 41 (19.8\%; 95\% CI:14.3-25.3) in the the preintervention group to 101 (52.1\%; $95 \%$ CI:45.0-59.2) in the postintervention group( $p$-value< 0.0001 ). Doctor's experience, shift or triage urgency did not influence compliance to the guideline. Secondary outcome measures did not significantly differ between the pre- and post-intervention group.

Conclusion Implementation of the guideline has lead to a significant higher frequency of correct assessment of UTI in young children. We could not identify determinants at patient, process or professional level with significant influence on successful implementation.

\section{DIFFERENCE BETWEEN ENTEROVIRUS AND HUMAN PARECHOVIRUS INFECTIONS IN YOUNG CHILDREN WITH SEPSIS-LIKE ILLNESS}

doi:10.1136/archdischild-2012-302724.0265

${ }^{1} E P$ De Jong, ${ }^{2} \mathrm{MGA}$ Van den Beuken, ${ }^{3} \mathrm{EPM}$ Van Elzakker, ${ }^{1} \mathrm{AJ}$ Sprij, ${ }^{4} \mathrm{FJ}$ Walther, ${ }^{1} \mathrm{~F}$ Brus. 'Juliana Children's Hospital, HAGA Hospital, The Hague; 'Paediatrics, Van WeelBethesda Hospital, Dirksland; 'Medical Microbiology, HAGA Hospital, The Hague; 4Paediatrics, Division of Neonatology, Leiden University Medical Center, Leiden, The Netherlands

Introduction Enterovirus (EV) or Human Parechovirus (HPeV) infections are common causes of sepsis-like illness in young children. We investigated differences in incidence, clinical characteristics and management of $\mathrm{EV}$ and $\mathrm{HPeV}$ infections among young children with sepsis-like illness.

Methods In 2008158 children under 36 months of age presenting with sepsis-like symptoms at the Juliana Children's Hospital in The Hague, the Netherlands, were evaluated in a prospective observational study. All received a sepsis work up including white cell count, CRP, blood culture and urine screening. Cerebrospinal fluid (CSF) was collected on clinical indication. EV or HPeV DNA was detected by PCR in plasma and/or CSF. Urine cultures were performed when urine screening was positive. 10 children with urinary tract infection were excluded. Data of the remaining 148 children were analysed.

Results EV/HPeV PCR was performed in 122/148 children: 45 (37\%) were EV positive and 22 (18\%) HPeV positive. The most prominent difference between children with $\mathrm{EV}$ and $\mathrm{HPeV}$ was age $\mathrm{HPeV}$ was solely diagnosed in children under 126 days of age. Clinical characteristics at presentation did not differ. Children with $\mathrm{HPeV}$ had lower leukocyte counts and lower CRP values. No difference in clinical management was found between $\mathrm{EV}$ and $\mathrm{HPeV}$ positive children.

Conclusion Sepsis-like illness due to $\mathrm{EV}$ and $\mathrm{HPeV}$ infection is common in young children, and appeared in $37 \%$ and $18 \%$ of cases respectively. $\mathrm{HPeV}$ occurs in younger children and causes less elevation of infectious parameters than $\mathrm{EV}$ infection. All other clinical characteristics are similar. Clinical management does not differ.

\section{THE ASSOCIATION BETWEEN RESPIRATORY TRACT UREAPLASMA UREALYTICUM COLONIZATION AND SEVERE RETINOPATHY OF PREMATURITY IN PRETERM INFANTS $\leq 1250 \mathrm{G}$}

doi:10.1136/archdischild-2012-302724.0266

${ }^{1} \mathrm{R}$ Ozdemir, ${ }^{1} \mathrm{FN}$ Sari, ${ }^{2 Z}$ Ozen Tunay, ${ }^{3} \mathrm{O}$ Erdeve, ${ }^{3} \mathrm{FE}$ Canpolat, ${ }^{3} \mathrm{SS}$ Oguz, ${ }^{3} \mathrm{~N}$ Uras, ${ }^{3} U$ Dilmen. ${ }^{1}$ Neonatology; ${ }^{2}$ Pediatric Ophthalmology; ${ }^{3} Z$ ekai Tahir Burak Maternity Teaching Hospital, Ankara, Turkey

Background and Aim To evaluate the association between respiratory tract Ureaplasma urealyticum $(U u)$ colonization and development of retinopathy of prematurity (ROP) requiring treatment.

Methods The infants with birthweight $\leq 1250 \mathrm{~g}$ born in a third level neonatal intensive care unit between March 2009 and May 2010 were prospectively identified. Nasopharyngeal swabs for $U u$ colonization were taken in postnatal first 3 days. Culture positive patients were reevaluated on the $12^{\text {th }}$ day by nasopharyngeal swabs for $U u$. The primary outcome was to define whether there was an association between respiratory tract $U u$ colonization and severe $\mathrm{ROP}$ requiring treatment. Independent samples t-test or Mann whitney $U$ test was used to compare continuous variables and $\mathrm{Chi}$ square test or Fisher's exact test for categorical variables. Multivariate (backward) logistic regression analysis was performed to simultaneously measure the influence of the independent variables with ROP as the dependent variable.

Results Twenty-five (12.1\%) infants developed severe ROP requiring treatment among 206 infants who underwent ROP screening. Mean birthweight and gestational age of total cohort were $1013 \pm 159 \mathrm{~g}$ and 27.9 \pm 1.6 weeks, respectively. Multivariate analysis demonstrated that birthweight (OR: 0.64 (95\% Cl 0.47-0.88); $\mathrm{p}=0.006)$, duration of mechanical ventilation (OR: $1.17(95 \%$ Cl 1.06-1.28); $\mathrm{p}=0.001$ ), premature rupture of membrane $>18 \mathrm{~h}$ (OR: 3.83 (95\% Cl 1.2-12.2); $\mathrm{p}=0.02$ ) and Uu positivity in both cultures (OR: 5.02 (95\% Cl 1.8-13.9); $\mathrm{p}=0.002$ ) were independent risk factors for the development of severe ROP requiring treatment.

Conclusions Respiratory tract colonization with $U u$ was independently associated with severe ROP requiring treatment.

\section{PROMOTING EARLY-LIFE IMMUNE DEVELOPMENT BY PERINATAL ADMINISTRATION OF PROBIOTICS TO PREGNANT/LACTATING MICE: OPTIMAL TIME WINDOW FOR INTERVENTION}

doi:10.1136/archdischild-2012-302724.0267 